Martinescu Alina ${ }^{1}$, Franciuc Irina ${ }^{2}$, Aftenie Loredana-Mariana ${ }^{2}$

\title{
HLA typing in autoimmune diseases
}

'Medical Genetics Department, Faculty of Medicine, 'Ovidius" University, Constanta, Romania

${ }^{2}$ Cell Biology Department, Faculty of Medicine, 'Ovidius" University, Constanta, Romania

\begin{abstract}
Genome scan, linkage and association studies have identified $\mathrm{CMH}$ genes in the genetic determinism of most autoimmune diseases, affecting approximately $5 \%$ of the population.

The susceptibility conferred by HLA alleles does not influence the development of autoimmunity in general, but rather the probability of some disorders.

From extremely high number of known HLA alleles, less than 30 are associated with diseases, and fewer than 10 are involved in the strongest associations.

The aim of this study was to determine HLA genes in autoimmune diseases.

The method used for the assignment of HLA alleles was molecular genotyping, primarily by the sequence specific oligonucleotide hybridization method (SSO), and when required, by the sequence specific primers method (SSP).

Molecular genotyping was performed in 282 cases of diabetes mellitus type 1, Hashimoto thyroiditis, Graves' disease and ankylosing spondylitis.

HLA typing in patients with autoimmune diseases is important for determining prognosis and genetic counseling. In ankylosing spondylitis HLA typing is used for differential diagnosis. In such studies it's important to be aware that there is a particular HLA gene expression depending on the geographic area.
\end{abstract}

Keywords: autoimmune diseases, major histocompatibility complex, risk alleles

\section{Martinescu Alina}

University “'Ovidius “, Faculty of Medicine, Constanta

1 Universitatii Blv, 900437 Constanta

$+40-0241605014$

Medical Genetics Department

Email: alinamartinescu@yahoo.co.uk

\section{Introduction}

Hundreds of diseases were tested for association with HLA. More than 50 statistical significant associations of HLA genes with different diseases are found so far [1].

Genome scan, linkage and association studies have identified $\mathrm{CMH}$ genes in the genetic determinism of most autoimmune diseases, affecting approximately $5 \%$ of the population [1].

$\mathrm{CMH}$ contribute at least $30 \%$ of familial aggregation in insulin-dependent diabetes mellitus, systemic lupus erythematous and rheumatoid arthritis $[1,2]$.

The association between a particular HLA allele and a disease is expressed as relative risk for the disease given the presence of particular HLA alleles (Table I).

It should be noted, however, that the same HLA allele may provide protection for a disease and at the same time, to confer susceptibility to another - for example alleles DQA1 * 0102, DQB1 * 0602 associated to HLA-DR2 are rare in patients with diabetes type 1 , but is associated with multiple sclerosis $[1,2]$.

It seems, therefore, that the susceptibility conferred by HLA alleles does not influence the development of autoimmunity in general, but rather the probability of some disorders.

From extremely high number of known HLA alleles, less than 30 are associated with diseases, and fewer than 10 are involved in the strongest 
associations.

Some researchers believe that these associations are conserved haplotypes that existed millions of years - known as ancestral haplotypes (for example haplotype A1, B8, DR3) [1]. From recombinant ancestral haplotypes results $75 \%$ of the haplotypes in the Caucasian population. Identifying the type of histocompatibility antigens present on cells of an individual, it is called HLA typing.

Table I. Several significant associations of increased risk HLA alleles for various diseases are. Adapted

\begin{tabular}{lcc}
\multicolumn{2}{c}{ from Goldsby et al., Immunology 5 th ed. 2003} \\
\hline \multicolumn{1}{c}{ Disease } & HLA associated & The relative risk* \\
\hline Retinopathy "birdshot" & A29 & 150 \\
Ankylosing spondylitis & $B 27$ & 90 \\
Reiter syndrome & $B 27$ & 37 \\
Reactive arthritis (Yersinia, & $B 27$ & 18 \\
Salmonella, Gonococcus) & DR2 &
\end{tabular}

$D R 3 / 5 / 7, D Q 2 \quad 130$

$\begin{array}{lcc}\text { Sensitivity to gluten enteropathy } & D R 3 / 5 / 7, D Q 2 & 130 \\ & & 30 \\ \text { Insulin-dependent diabetes } & D R 4 / D R 3, & 20\end{array}$

mellitus DQ2/DQ8

Goodpasture syndrome

Myasthenia gravis

Rheumatoid arthriti

Multiple sclerosis

Systemic lupus erythematosus

Hashimoto's thyroiditis

* Relative risk is calculated by dividing the frequency of HLA in the patient population at its frequency in the general population.

$\begin{array}{cc}D R 2 & 16 \\ D R 3 & 10 \\ D R 4 & 10 \\ D R 2, D R 6 & 5 \\ D R 3 & 5 \\ D R 5 & 3\end{array}$

Material and method

During October 2008 - September 2011 HLA genotyping was performed in 282 cases registered at Molecular Laboratory of Medical Genetics Department for following diseases: 62 for diabetes mellitus type 1, 98 for Hashimoto thyroiditis, 112 for Graves' disease and 10 for ankylosing spondylitis.

The method used for the assignment of HLA alleles was molecular genotyping, primarily by the sequence specific oligonucleotide hybridization method (SSO), and when required, by the sequence specific primers method (SSP).

In the SSO method, polymorphic regions of genes of class I and II are amplified from genomic DNA by PCR reaction. Then, resulting amplicons are used in hybridization reaction with specific oligonucleotide probes, selected to detect regions with polymorphism. (See Figure $1 \mathrm{a}, \mathrm{b}$ ).
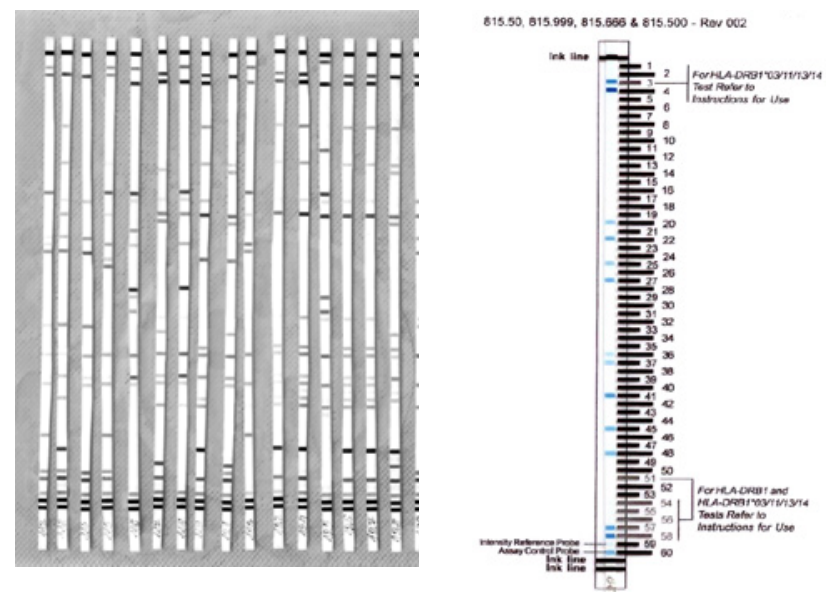

\section{Aim of study}

The aim of this study was to determine HLA genes in autoimmune diseases.

.


sequence-specific primers systems (SSP). DNA amplification occurs only if in genomic DNA it is a sequence perfectly complementary with primers. The presence or absence of PCR products of correct size is than analyzed by electrophoresis. (see Figure 3 and $4)$.

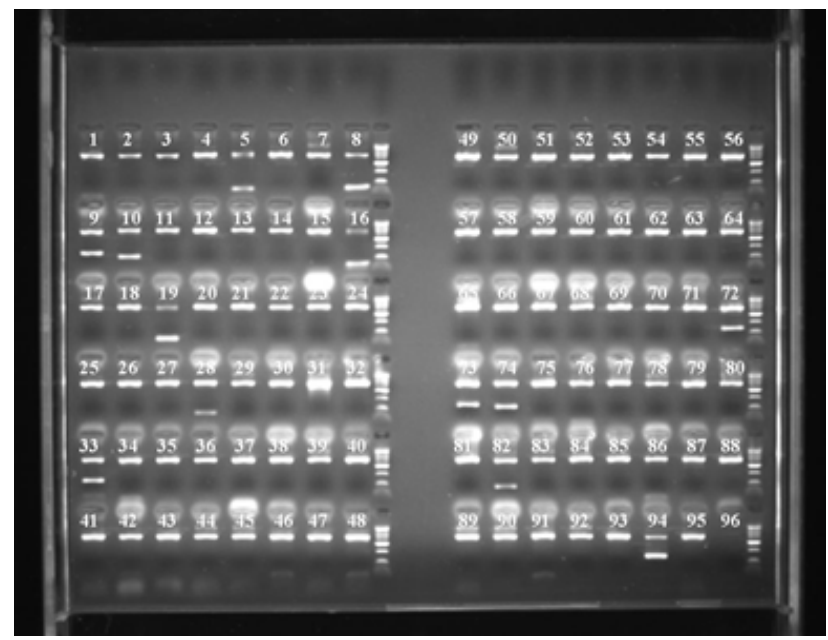

Figure3. Gel image photographed by a digital camera of the gel documentation system. Control lines are observed (black arrow) in all tracks except number 96 which is negative amplification control. That shows the presence of all amplification reactions (1-95). Lack of amplification products in position 96 shows no contamination. Specific lines $(5,8,9,10,16,19$, etc. open black arrow) indicate the presence of specific polymorphism in the HLA-DRBI gene structure. Weak nonspecific lines can be observed (white arrow) that are usually unused primers or primer dimmers.

Than gel photo is print and apply on gel documentation sheet. After a carefully examination of the gel are determined the positive tracks. They are marked on the form (Figure 4).

After establishing positive lines, alleles designation is made by using a computer program called UniMatch $^{\mathrm{TM}}$ (Figure 5). In the dialog is selected the type of determination used (e.g. High Resolution HLA-DRB1), patient data recorded and specificities found (positive lines on electrophoresis).

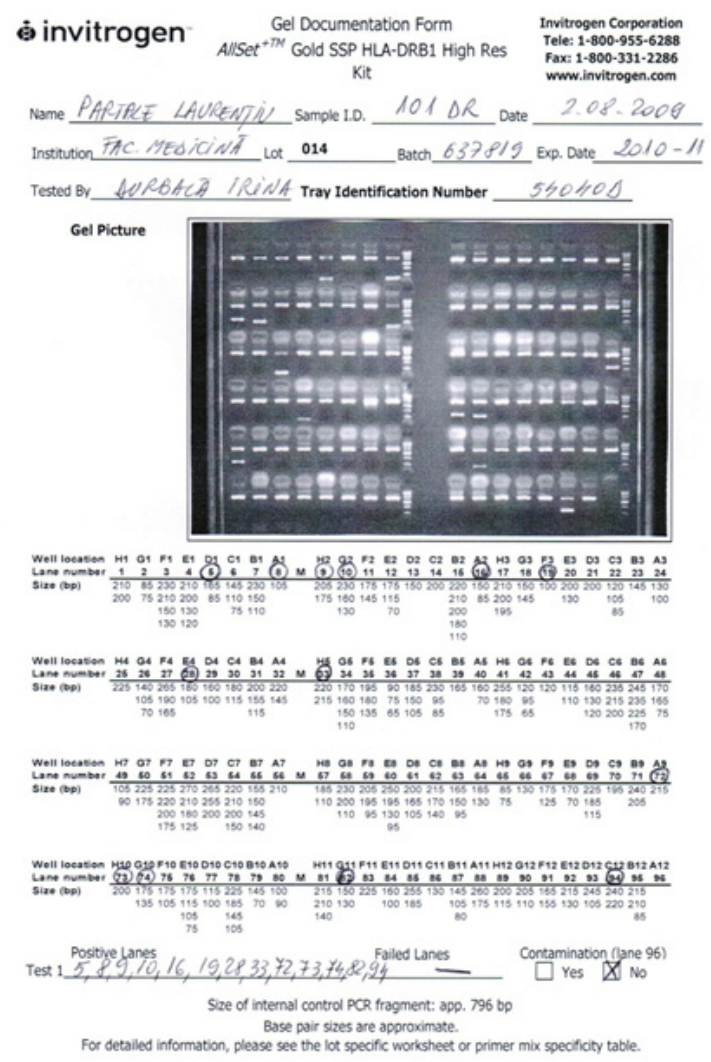

Figure 4. Gel documentation form. Gel photograph is attached by the form. Patient data and data about the kit used are note too. Tracks marked with specific

amplification, those were amplification failed and

presence or absence of contamination.

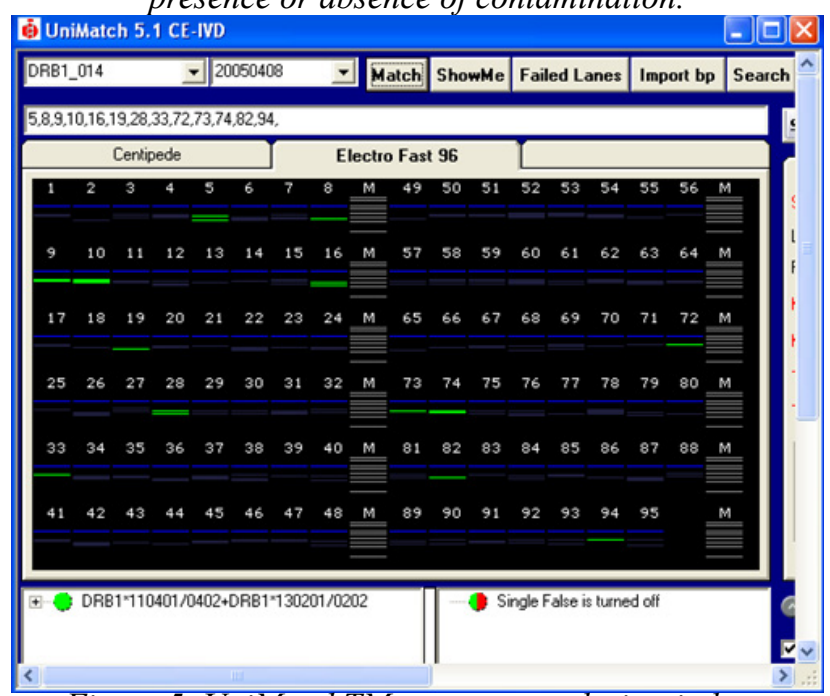

Figure 5. UniMatchTM program analysis window: determination of HLA-DRB1 High Resolution. The specificities marked as positive are: 5, 8, 9, 10, 16, 19, 28, $33,72,73,74,82,94$. HLA-DRB1 alleles are designated:

* 1104 and * 1302 


\section{Results and discussions}

In our laboratory were 282 HLA typing for the following diseases: diabetes mellitus type 1, Hashimoto thyroiditis, Graves' disease, celiac disease and ankylosing spondylitis.

From 62 patients with type 1 diabetes 95.1\% have one or both risk alleles DR3 or DR4, similar to those of other studies that have analyzed the HLA genes in 14168 children with type 1diabetes [1].

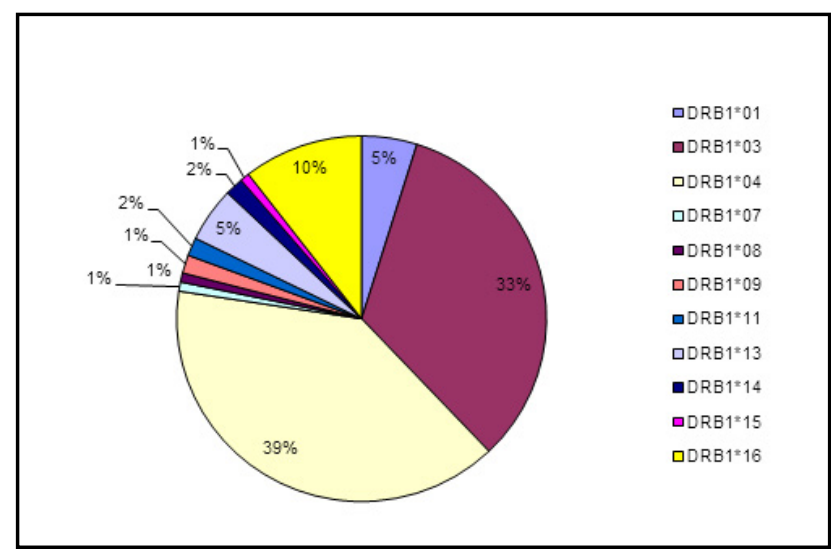

Figure 6. The frequency of HLA-DRB1alleles in patients with DMI

Frequency of genotype DR3 / 4 (30.64\%) was slightly lower than the frequencies of $36-38 \%$ in the studies mentioned, but was slightly higher than in other studies of type 1 diabetes from Romania $[1,2]$, who reported a frequency of $20.75 \%$ for this genotype. Non DR3-nonDR4 genotype frequency was lower in our study (4.9\%) than in other studies from Romania, in which had found a frequency of $10.8 \%$ for this genotype. Results are similar for DQ due to linkage disequilibrium between DR3 - DQ2 and DR4 - DQ8.

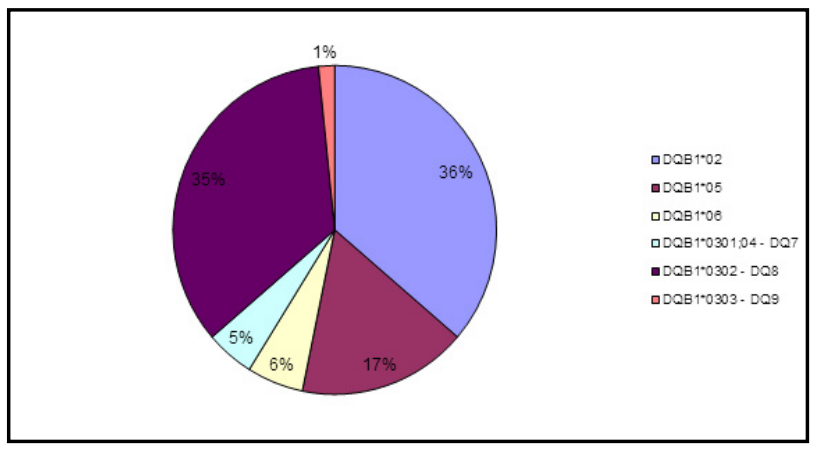

Figure 7. The frequency of $H L A-D Q B 1$ alleles in patients with DM1

4 patients with DM1 had also celiac disease. We found 3 patients with DR3-DQ2 and one with DR4 - DQ8. 95\% of patients with celiac disease have one of the genes HLA DQ2 or DQ8. The association between the two diseases can be explain by common susceptibility genes conferred by HLA class II.

In Caucasians, the risk alleles of HLA class II genes are considered HLA-DRB $1 * 04, * 05$ (divided into $* 11$ and $* 12$ allele groups) and inconstant HLADRB1*03 for Hashimoto thyroiditis [3].

In our study from Dobrogea, besides HLADRB1*11 (DR5) risk alleles, we found also HLADRB $1 * 03$ and HLA-DRB1*16 predisposing for Hashimoto thyroiditis.

From 98 patients with HT only 8 associated diabetes mellitus type 1 and 4 rheumatoid arthritis. These associations are expected because they share the same HLA-DRB1 risk alleles. However 74.5\% of cases are not associated with other autoimmune diseases.

In a multicenter study from England, only $14 \%$ of patients with Hashimoto thyroiditis associate other autoimmune diseases, and the most frequent was rheumatoid arthritis [3].

In most studies, HLA-DRB1*03 alleles are predisposing to Graves' disease in Caucasians [1]. In our study both HLA-DRB1*03 and *04 are predisposing to Graves' disease. From 112 patients only 10 associated rheumatoid arthritis and all had HLA- DRB1*04. 9 patients with GD associated DM 1 and all had HLA-DRB $1 * 03$ or *04.

In a study of 2791 Caucasian with Graves' disease, only $9.67 \%$ had other autoimmune diseases 
of witch $15.3 \%$ had rheumatoid arthritis [1,2].

Only 10 patients with ankylosing spondylitis were typing HLA. 3 of them were homozygous and 7 heterozygous for HLA B*27. There are described 40 allelic variants but only HLA B*27:05 is present in 95\% English caucasians. Subtype HLA B*27:06/09 have a low penetrance.

\section{Conclusions}

HLA typing in patients with autoimmune diseases is important for determining prognosis and genetic counseling. In ankylosing spondylitis HLA typing is used for differential diagnosis.

If the predisposition for the diseases is determined by all the alleles of the group, than a low resolution HLA typing is performed. But if it is necessary to identify exactly the allele from a group, than a high resolution HLA typing is indicated.

In such studies it's important to be aware that there is a particular HLA gene expression depending on the geographic area.

\section{References}

1. Guja C. (2006) Particularitati genetice la DZ tip 1 la populatia din Romania. In: Guja C. Factori genetici implicati in etiopatogenia diabetului zaharat tip 1. The Publishing House of the Romanian Academy, Bucharest. 223-31.

2. Guja C, Guja L, Nutland S, Rance H, Sebastien M, Todd JA. \& Ionescu-Tirgoviste C. (2004) Type 1 diabetes genetic susceptibility encoded by HLA DQB1 genes in Romania. $J$ Cell $\mathrm{Mol}$ Med.;8(2), 249-56.

3. Boelaert K, Newby PR \& Simmonds MJ. ( 2010) Prevalence and relative risk of other autoimmune diseases in subjects with autoimmune thyroid disease. Am J Med.;123(2), 183 